\title{
Notulæ ad Plantas Asiaticas Orientales. \\ (Continued from p. 71.) \\ Auctore
}

J. Matsumura.

Lysimachia lineariloba, Hook. et Arn. Bot. Beech. Voy. p. 268 ; Duby in DC. Prodr. VIII. p. 61 ; Sieb. et Zucc. Fl. Jap. Fan. Nat. no. 472 ; Miq. Prol. p. 285 ; Franch. et Sav. Enum. Pl. Jap. I. p. 300 et II. p. 431 ; Forbes et Hemsl. in Journ. Linn. Soc. XXVI. p. 53 ; Henry in Trans. Asiat. Soc. Jap. XXIV. Suppl. no. 584. Lysimachia lubinioides, Sieb. et Zucc. Fl. Jap. Fam. Nat. no. 471 ; Benth. et Hook. Gen. Pl. II. p. 635 ; Miq. Prol. p. 285 ; Franch. et Sav. Enum, Pl. Jap. I. p. 292. L. spathulata, Klatt in $\Lambda$ bhandl. Naturw. Ver. Hamb. IV. t. 18 ; Hillebr. Fl. Hawai. Isl. p. 285. L. mauritiana, Lam. Lubinia spathulata, Vent; Duby in DC. Prodr. VIII. p. 60. Soomoku-zusetsu III. t. 57.

Hab. in Liukiu: ins. Okinawa (Y. Tashiro), ad Nana ejusdem ins. (J. Matsumura); in Formosa orientali, inter Myokoosha et Taikookoo (K. Miyake); ins. Botel-Tobago (K. Miyake).

Lysimachia cletbroides, Duby in DC. Prodr. VIII. p. 61 ; Miq. Prol. p. 284 ; Franch. et Sav. Enum. Pl. Jap. I. p. 301.

Lus. longebracteata, bracteis foliaceis pedicellos bis-quinquies superantibus.

Hab. in Japonia : ins. Yezo (Bochmer); prov. Shinano, in monte Usui (J. Matsumura et R. Yatabe).

Lysimachia candida, Lindl.; Hance, Journ. Bot. 1877, p. 357; Tranchet, Pl. David. p. 200 ; Maxim. in Bull. Soc. Nat. Mosc. 1879, p. 30 ; Klatt in Abhandl. Naturw. Ver. Hamb. IV. 4 abt. t. 1. fig. dext.; Forbes et Hemsl. in Journ. Linn. Soc, XXVI. p. 48. Lysimachia leucantha, Miq. Prol. p. 285 ; Franch. et Sav. Enum. Pl. Jap. I. p. 301 et II. p. 431 ; Soomoku-zusetsu III. t. 63.

Hab. in Formosa: Shin-ei-shoo, Tailan (C. Owatari); in Japonia : prope urbem Tōôyō (T. Terasaki), ad Usa, prov. Buzen (J. Matsumura et R. Yatabe). 
Lysimachia sikokiana, Miq. Prol. (1867) p. 285 ; Franch. et Sav. Enum. Pl. Jap. I. p. 302. Lysimachia Fonumgraecum, Hance in Journ. Bot. (1877) p. 355 ; Forbes et Hemsl. in Journ. Linn. Soc. XXVI. p. 51. L. simulans, Hemsl.; Forbes et Hemsl. in Journ. Linn. Soc. XXVI. p. 57 ; Henry in Trans. Asiat. Soc. Jap. XXIV. Suppl. p. 57, no. 583; Chi wu ming shi tu kao XXV. fol. 74 ; Soomoku-zusetsu III. t. 65 ; Honzoozufu XI. fol. 13, fig. sinist.

Hab. in Formosa : Kelung (T. Makino), inter Ulai et Lahoo (K. Miyake); Liukiu: ins. Yonakuni (S. Tanaka, no. 405), ins. Okinawa (J. Matsumura, S. Tanaka, no. 174, Y. Tashiro), ins. Yaeyama (Y. Tashiro); in Japonia : prov. Tosa (S. Yano).

Lysimachia japonica, Thunb. Fl. Jap. p. 83; Ic. Pl. Jap. t. 16 . Duby in DC. Prodr. VIII. p. 67 ; Sieb. et Zucc. Fl. Jap. Fam. Nat. no. 469 ; Klatt in Abhandl. Naturw. Ver. Hamb. IV. 4 abt. p. 34, t. 19 ; Hook. f. Fl. Brit. Ind. III. p. 505 ; Kurz. in Journ. Bot. 1873, p. 193 ; Hance in Journ. Bot. 1877, p. 357 ; Forbes et Hemsl. in Journ Linn. Soc. XXVI. p. 53 ; Henry in Trans. Asiat. Soc. Jap. XXIV. Suppl. p. 57, no. 582. Lysimachia ferruginea, Edgew. in Trans. Linn. Soc. XX. p. 83 ; S; Moore in Journ. Bot. 1878, p. 138; Hance in Journ Bot, 1880, p. 299 ; Klatt in Abhandl. Naturw. Ver. Hamb. IV. abt. 4, p. 35, t. 20.

Hab. in Liukiu : ins. Okinawa (S. Tanaka, no. 207).

Anagallis arvensis, L. Sp. Pl. ed. II. p. 211 ; Duby in DC. Prodr. VIII. p. 69 ; Sieb. et Zucc. Fl. Jap. Fam. Nat. no. 473. Hook. f. Fl. Brit. Ind. III. p. 506 ; Franch. et Sav. Enum. Pl. I. p. 303 ; Forbes et Hemsl. in Journ. Linn. Soc. XXVI. p. 59 ; Henry in Trans. Asiat. Soc. Jap. XXIV. Suppl. p. 57, no. 585. Anagallis phoenicea, Thunb. Fl. Jap. p. 88.

Harb. in Liukiu : ins. Miyako (Y. Tashiro), ins. Okinawa (Y. Tashiro); in Formosa: Pachina (Honda et Niinami, no. 34), Pehsoaton (C. Owatari); Shinchiku (Hiraoka), Suiteilyau (C. Owatari).

Samolus Valerandi, L. Sp. ed. 2. p. 243; Duby in DC. Prodr. VIII. p. 73 ; Hook. f. Fl. Brit. Ind. III. p. 506 ; A. Gray Syn. Fl. N. Am. II. 1. p. 64 ; Forbes et Hemsl. in Journ. Linn. Soc. XXVI. p. 59. S. floribundus, H. B. Kunth, Max. in Mel. Biol. XII. p. 492. S. Valerandi, var. americanus, A. Gray 1.c. 64 .

Hab. in insula Yezo: Zenibako (K. Miyabe), in paludosis ad Joozankei (J. Matsumura); ad Ichinomiya, prov. Kazusa (J. Matsumura). 
Glaux maritima, T. Sp. ed. 2. p. 301 ; Ledeb. Fl. Ross. III. p. 23 ; Duby in DC. Prodr. VIII. p. 59; Bunge Enum. Pl. Chin. no, 300; Maxim. Prim. Fl. Amur. p. 192 et Ind. Fl. Pek. p. 474 ; Schmidt. Fl. Sachal. p. 159 ; Herd. in Act. Hort. Petrop, I. 2. p. 411 ; Franch. et Sav. Enum. Pl. Jap. II. p. 430 ; Franchet, Pl. David. p. 201 ; Hook. f. Fl. Brit. Ind. III. p. 505 ; A. Gray, Syn. Fl. N. Am. II. 1. p. 63 ; Forbes et Hemsl. in Journ. Tinn. Soc. XXVI. p. 59.

Hab. insula Yezo: Mororan (R. Yatahe, anno 1878 ; J. Matsumura anno 1899), prov. Nemuro, ad Toobnto ejusdem insule (K. Miyabe, anno 1884), Fukuyama, prov. Ojima ejusdem insulke (K. Miyabe et Y. Tokubuchi anno 1890).

(To be continued.)

\title{
Plantæ Sinenses Yoshianæ.
}

\author{
V. \\ Auctore \\ Tokutaro Ito.

\section{CRUCIFER $A$.}

Sisymbrium Sophia, Linn. Sp. Pl. ed. 1, p. 659 ; Eng. Bot. t. 963 ; DC. Syst. II, p. 474, et Prodr. I, p. 193 ; Ledeb. Fl. Alt. III, p. 135, et Fl. Ross. I, p. 180 ; Bunge, Enum. Pl. Chin. Bor. p. 6 ; Hook. f. et Anders. in Hook. f. Fl. Brit. Ind. I, p. 150 ; Regel et Til. Fl. Ajan. p. 60 ; Regel, in Bull. Soc. Nat. Mosc. 1861, 3, p. 203 ; Maxim. Primit. Fl. Amur. pp. 469 (Ind. Fl. Pekin.), 480 (Ind. Fl. Mongol.), Enum. Pl. Mongol. p. 59, Fl. Tangut. p. 60, et in Act. Hort. Petrop. XI, p. 53; Debeaux, Fl. Tehef. p. 26 ; Nyman, Consp. p. 43 ; Franch. Pl. David. I, p. 36 ; Forbes et Hemsl. in Journ. Linn. Soc. XXIII, p. 46 ; Dur. et Schinz, Consp. Fl. Afr. I, 2, p. 98.

Hab. in provincia Shantung: Yantai prope urbem Tshifu, 14 Aprilis 1898, flor. cum fruct. immat. (T. Takagaki!). 